\title{
Digital Chemistry
}

\author{
Lutvo Kurić \\ Institute of Economics, University of Sarajevo, Trg Oslobođenja 1, Sarajevo, \\ Bosnia and Herzegovina \\ E-mail address: lutvokuric@yahoo.com
}

\begin{abstract}
The subjects of our research are program lawfulness, cybernetic lawfulness, and informational lawfulness in molecule sequence in the nature. In the science, one question has been present for a long time, that is, if there is one unique common connection that links all phenomena in the cosmos. The doubt is, if the periodical in the biochemical process is only a physical-chemical matter of objective material relationship or maybe a matter of numbers and mathematics. With the goal to find the answers on some of those questions, we have made a decision to do a research on, if in the nature exists such one unique mathematical array and one mathematical lawfulness which could be valid for all phenomena in chemistry and physics.
\end{abstract}

Keywords: Digital chemistry; chemical compounds; codes in chemistry; code speed of light; quantum physics

\section{INTRODUCTION}

What we did is the following: We translated the physical and chemical parameters from the language of biochemistry into the digital language of programmatic, cybernetic and information principles. This we did by using the adequate mathematical algorithms. By using chemical-information procedures, we calculated the numerical value for the information content of molecules. What we got this way is the digital picture of the phenomenon of biochemistry. These digital pictures reveal to us a whole new dimension of this science. They reveal to us that the biochemical process is strictly conditioned and determined by programmatic, cybernetic and information principles.

Within the digital pictures in biochemistry, the physical and chemical parameters are in a strict compliance with programmatic, cybernetic and information principles. Each bar in the protein chain attracts only the corresponding amino acid, and only the relevant amino acid can be positioned at certain place in the chain. Each peptide chain can have the exact number of amino acids necessary to meet the strictly determined mathematical conditioning. It can have as many atoms as necessary to meet the mathematical balance of the biochemical phenomenon at certain mathematical level, etc. The digital language of biochemistry has a countless number of codes and analogue codes, as well as other information content. These pictures enable us to realize the very essence of functioning of biochemical processes. 


\section{RESULTS}

The herewith discussed research results show that the process of sequencing in macromolecules is conditioned and determined not only through biochemical, but also through cybernetic information principles.

We would particularly like to stress here that the chemistry, well as biochemical information in a broader sense of the word, is determined and characterized by very complex cybernetic and information principles. The constantans in those principles are: the number of atoms and molecules, atomic numbers, atomic weight, physical and chemical parameters, even and odd values, codes and analogue codes, standard deviations, frequencies, primary and secondary values, and many other things. How functioning of biochemistry is determined through cybernetic information principles, will be discussed further in this text.

\section{Algorithm:}

INPUT $=$ Natural numbers in-group of numbers from $\mathrm{X}$ to $\mathrm{Y}$ OUTPUT $=$ Arithmetical expression for code and decode of sequencing of phenomena

Expression for output is following:

$$
\{[(\mathrm{SA}(\mathrm{R} 1,2,3, \mathrm{n})-(\mathrm{SB}(\mathrm{R} 1,2,3, \mathrm{n}))]+(\mathrm{A} \times \mathrm{B})\}=\mathrm{X}
$$

A \& $\mathrm{B}=$ Constants for sequencing of molecules in Nature,

$\mathrm{S}=$ Groups of numbers in arithmetic expressions $1,2,3, \mathrm{n}$, $\mathrm{R}(1,2,3, \mathrm{n})=$ Arithmetical expression for the phenomena $1,2,3, \mathrm{n}$,

$\mathrm{X}=$ Arithmetical expression for the code matrix in nature

Solution to this formula is: $A=7 ; B=19 ; \mathrm{X}=(7 \times 19 \times 7)$;

Consequently the most possible combination for sequencing of phenomena in nature is the one in which input factors are 19 and 7 , and number $(7 \times 19 \times 7)$ - output result.

\section{Silver monohydride}

\section{Example 1}

AgH silver monohydride 13967-01-6

Atomic numbers

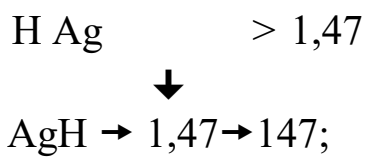




$$
\begin{gathered}
\mathrm{AgH} \rightarrow 147 \\
\{[(\mathrm{~S} 7(147) \times 19)-(\mathrm{S} 19(147) \times 7)]+[(7 \times 19)]\}=(7 \times 19 \times 7) \\
\mathrm{S} 7(147)=(141+142+143+144+145+3146+147)=1008 \\
\mathrm{~S} 7(147)=1008 \\
\mathrm{~S} 19(147)=(129+130+131+132 \ldots+.147)=2622 \\
\mathrm{~S} 19(147)=2622 \\
\{1008 \times 19)-(2622 \times 7)]+[(7 \times 19)]\}=(7 \times 19 \times 7) \\
\{19152-18354+(7 \times 19)\}=(7 \times 19 \times 7)
\end{gathered}
$$

\section{Result 1:}

$\mathrm{H}=$ Code 19152;

$\mathrm{Ag}=$ Code 18354;

\section{Example 2.}

AgH silver monohydride 13967-01-6

$$
\begin{gathered}
\text { Atomic numbers } \\
\text { AgH } \stackrel{\downarrow}{\rightarrow} \text { 47,1 } \\
\text { AgH }>47,1>471 ; \\
\operatorname{AgH}>471 ; \\
\{[(\mathrm{S} 7(471) \times 19)-(\mathrm{S} 19(471) \times 7)]+[(7 \times 19)]\}=(7 \times 19 \times 7) ; \\
\mathrm{S} 7(471)=3276 ; \\
\mathrm{S} 19(471)=8778 ; \\
\{3276 \times 19)-(8778 \times 7)]+[(7 \times 19)]\}=(7 \times 19 \times 7) ; \\
\{62244-61446+(19 \times 7)\}=(7 \times 19 \times 7) ;
\end{gathered}
$$

\section{Result 2:}

$\mathrm{H}=$ Code 62244 ;

$\mathrm{Ag}=$ Code 61446; 
Correlaton of silver monohydride and aluminum hydride:

$$
\begin{aligned}
(62244-19152) & =(61446-18354) \\
(62244+18354) & =(61446+19152)
\end{aligned}
$$

\section{Aluminum hydride}

\section{Example 1}

\section{Atomic numbers}

AlH aluminum hydride 13967-22-1

$$
\begin{gathered}
\text { HAl } \underset{\downarrow}{\downarrow} \rightarrow 1,13 \\
\mathrm{HAl} \rightarrow 1,13 \rightarrow 113 ; \\
\{[(\mathrm{S} 7(113) \times 19)-(\mathrm{S} 19(113) \times 7)]+[(7 \times 19)]\}=(7 \times 19 \times 7) ; \\
\mathrm{S} 7(113)=770 ; \\
\mathrm{S} 19(113)=1976 ; \\
\{770 \times 19)-(1976 \times 7)+[(7 \times 19)]\}=(7 \times 19 \times 7) ; \\
(14630-13832+133)=(7 \times 19 \times 7) ;
\end{gathered}
$$

Result:

$\mathrm{H}=$ Code 14630 ;

$\mathrm{Al}=$ Code 13832;

Correlation:

$($ Code $19152-$ Code 14630$)=($ Code $18354-$ Code 13832$)$

$$
\begin{gathered}
\text { DET }=-3608556 ; \\
3608556=[(7 \times 19 \times 7) \times \mathrm{Y}] ;
\end{gathered}
$$




\section{Example 2}

\section{Atomic numbers}

AlH aluminum hydride 13967-22-1

$$
\begin{gathered}
\mathrm{AlH} \underset{\downarrow}{\downarrow} \rightarrow 13,1 \\
\mathrm{AlH} \rightarrow 13,1 \rightarrow 131 ; \\
\{[(\mathrm{S} 7(131) \times 19)-(\mathrm{S} 19(131) \times 7)]+[(7 \times 19)]\}=(7 \times 19 \times 7) ; \\
\mathrm{S} 7(131)=896 ; \\
\mathrm{S} 19(131)=2318 ; \\
\{896 \times 19)-(2318 \times 7)+[(7 \times 19)]\}=(7 \times 19 \times 7) ; \\
{[(17024-16226+(19 \times 7)]=(7 \times 19 \times 7) ;}
\end{gathered}
$$

\section{Result:}

$\mathrm{Al}=$ Code $17024 ; \mathrm{H}=$ Code $16226 ;$

\section{Correlation:}

$($ Code $62244-$ Code 17024$)=($ Code $61446-$ Code 16226$)$

\section{Water}

$\mathrm{H}_{2} \mathrm{O}$ water 7732-18-5

\section{Example 1}

\section{Atomic numbers}

$\mathrm{H}_{2} \mathrm{O}$ water $\mathrm{HOH}$

$$
\begin{gathered}
\mathrm{HOH} \underset{\downarrow}{\downarrow} \rightarrow 1,8,1 \\
\mathrm{HOH} \rightarrow 1,8,1 \rightarrow 181 ; \\
\{[(\mathrm{S} 7(181) \times 19)-(\mathrm{S} 19(181) \times 7)]+[(7 \times 19)]\}=(7 \times 19 \times 7) ; \\
\mathrm{S} 7(181)=1246 ;
\end{gathered}
$$




$$
\begin{gathered}
\mathrm{S} 19(181)=3268 \\
\{1246 \times 19)-(3268 \times 7)+[(7 \times 19)]\}=(7 \times 19 \times 7) \\
{[(23674-22876+(19 \times 7)]=(7 \times 19 \times 7)}
\end{gathered}
$$

Result:

$\mathrm{HOH}=$ Code 23674 and 22876;

\section{Example 2}

Atomic numbers

AlH aluminum hydride 13967-22-1

$$
\begin{gathered}
\mathrm{AlH} \underset{\downarrow}{\downarrow} \rightarrow 13,1 \\
\mathrm{AlH} \rightarrow 13,1 \rightarrow 131 ; \\
\{[(\mathrm{S} 7(131) \times 19)-(\mathrm{S} 19(131) \times 7)]+[(7 \times 19)]\}=(7 \times 19 \times 7) ; \\
\mathrm{S} 7(131)=896 ; \\
\mathrm{S} 19(131)=2318 ; \\
\{896 \times 19)-(2318 \times 7)+[(7 \times 19)]\}=(7 \times 19 \times 7) ; \\
{[(17024-16226+(19 \times 7)]=(7 \times 19 \times 7) ;}
\end{gathered}
$$

Result:

$\mathrm{Al}=$ Code $17024 ; \mathrm{H}=$ Code 16226

\section{Correlation:}

$($ Code $62244-$ Code 17024$)=($ Code $61446-$ Code 16226$)$

etc. 


\section{PEPTIDE CHAIN}

CYS and GLY

\begin{tabular}{|c|c|c|c|c|c|c|}
\hline & Cys & & & Gly & & \\
\hline & $\begin{array}{l}\mathrm{C}_{3} \mathrm{H}_{7} \\
\mathrm{~N}_{2} \mathrm{OS}\end{array}$ & & & $\begin{array}{l}\mathrm{C}_{2} \mathrm{H}_{5} \\
\mathrm{~N}_{2} \mathrm{O}\end{array}$ & & \\
\hline $\mathbf{K}$ & & y & $\boldsymbol{k}$ & & $y$ & \\
\hline $\mathrm{HNH}$ & & $\mathrm{COOH}$ & $\mathrm{HNH}$ & & $\mathrm{COOH}$ & \\
\hline$\downarrow$ & & y & $\boldsymbol{k}$ & & $\downarrow$ & \\
\hline $\mathrm{HNH}$ & & \multicolumn{2}{|c|}{$\mathrm{CONH}$} & & $\mathrm{COOH}$ & $+\mathrm{HOH}$ \\
\hline$\downarrow$ & & \multicolumn{2}{|r|}{$\downarrow$} & & $\downarrow$ & $\downarrow$ \\
\hline $1,7,1$ & & \multicolumn{2}{|c|}{$6,8,7,1$} & & $6,8,8,1$ & $1,8,1$ \\
\hline$\downarrow$ & & \multicolumn{2}{|r|}{$\downarrow$} & & $\downarrow$ & $\downarrow$ \\
\hline 171 & & \multicolumn{2}{|c|}{6871} & & 6881 & 181 \\
\hline & $y$ & \multicolumn{2}{|r|}{$\downarrow$} & $\boldsymbol{k}$ & $\boldsymbol{k}$ & $k$ \\
\hline & & \multicolumn{2}{|c|}{14104} & & & \\
\hline $\boldsymbol{K}$ & $\boldsymbol{k}$ & $\boldsymbol{k}$ & $y$ & y & $y$ & y \\
\hline $\begin{array}{c}931,93 \\
1,931\end{array}$ & $\begin{array}{c}931,93 \\
1,931\end{array}$ & \multicolumn{2}{|c|}{$931,139,931$} & 931 & $\begin{array}{c}931,931, \\
931\end{array}$ & $\begin{array}{c}931,93 \\
1,931\end{array}$ \\
\hline
\end{tabular}

$14104=(171+6871+6881+181)$

$14104=[(931+931+931)+(931+931+931)+(931+139+931)+931+(931+931+931)+$ $+(931+931+931)]$

$931=(7 \times 19 \times 7)$

PHE and VAL

\begin{tabular}{|c|c|c|c|c|c|}
\hline & Phe & & & Val & \\
\hline & $\begin{array}{l}\mathrm{C}_{9} \mathrm{H}_{11} \\
\mathrm{~N}_{2} \mathrm{O}\end{array}$ & & & $\begin{array}{c}\mathrm{C}_{5} \mathrm{H}_{11} \\
\mathrm{~N}_{2} \mathrm{O}\end{array}$ & \\
\hline $\boldsymbol{K}$ & & y & $\boldsymbol{K}$ & & $y$ \\
\hline $\mathrm{HNH}$ & & $\mathrm{COOH}$ & $\mathrm{HNH}$ & & $\mathrm{COOH}$ \\
\hline$\downarrow$ & & $y$ & $\boldsymbol{K}$ & & $\downarrow$ \\
\hline
\end{tabular}




\begin{tabular}{|c|c|c|c|c|c|}
\hline $\mathrm{HNH}$ & & $\mathrm{CONH}$ & & $\mathrm{COOH}$ & $+\mathrm{HOH}$ \\
\hline$\downarrow$ & & $\downarrow$ & & $\downarrow$ & $\downarrow$ \\
\hline $1,7,1$ & & $6,8,7,1$ & & $6,8,8,1$ & $1,8,1$ \\
\hline$\downarrow$ & & $\downarrow$ & & $\downarrow$ & $\downarrow$ \\
\hline \multirow[t]{3}{*}{171} & & 6871 & & 6881 & 181 \\
\hline & $y$ & $\downarrow$ & $\mathbf{k}$ & $\mathbf{K}$ & $\mathbf{K}$ \\
\hline & & 14104 & & & \\
\hline $\boldsymbol{k}$ & $\boldsymbol{k}$ & $\boldsymbol{K}$ & $y$ & $y$ & $y$ \\
\hline $\begin{array}{c}931,931,9 \\
31\end{array}$ & $\begin{array}{c}931,931, \\
931\end{array}$ & $931,139,931$ & 931 & $\begin{array}{c}931,931, \\
931\end{array}$ & $\begin{array}{c}931,93 \\
1,931\end{array}$ \\
\hline
\end{tabular}

$14104=(171+6871+6881+181) ;$

$14104=[(931+931+931)+(931+931+931)+(931+139+931)+931+(931+931+931)+$ $+(931+931+931)]$

$931=(7 \times 19 \times 7)$

etc.

\section{THE SPEED OF LIGHT}

Value:

$186282 \mathrm{mi} / \mathrm{s}$ (miles per secend)

$\downarrow$

Deconnection:

$186282 \rightarrow(1862$ and 82$)$

$$
1862=(7 \times 19 \times 7)+(7 \times 19 \times 7)
$$

\section{Code speed of light}

$\{[(\mathrm{S} 7(186282) \times 19)-(\mathrm{S} 19(186282) \times 7)]+[(7 \times 19)]\}=(7 \times 19 \times 7)$

$\mathrm{S} 7(186282)=1303953$ 


$$
\begin{gathered}
\mathrm{S} 19(186282)=3539187 \\
\{1303953 \times 19)-(3539187 \times 7)+[(7 \times 19)]\}=(7 \times 19 \times 7) \\
{[(24775107-24774309+(19 \times 7)]=(7 \times 19 \times 7)}
\end{gathered}
$$

Result:

Code 1 = 24775107; Code 2 = 24774309;

\section{Comment:}

$$
\begin{gathered}
(24775107 \times 24774309)=[(\mathrm{A} \times \mathrm{B} \times \mathrm{A}) \times \mathrm{Y}] \\
{[(7 \times 19 \times 7) \times \mathrm{Y}]=613786156326063} \\
\mathrm{Y}=659276215173
\end{gathered}
$$

Correlation of the speed of light and quantum physics

\begin{tabular}{|c||}
\hline The speed of light \\
$\downarrow$ \\
\hline $299.792,458$ \\
\hline \\
\hline 299792458 \\
\hline
\end{tabular}

Quantum physics

$$
\mathbf{a}_{\mathbf{0}}=\frac{\mathrm{n}^{2}}{\mathrm{mke}^{2}}=0.529 \times 10^{-10} \text { meters }
$$

Where:

$$
\begin{aligned}
& \mathrm{h}=\frac{\text { Planck's constant }}{2 \pi}=1.055 \times 10^{-34} \text { Joule-seconds } \\
& \mathrm{m}=\text { mass of electron }=9.109 \times 10^{-31} \text { kilograms } \\
& \mathrm{k}=\text { Coulomb's constant }=8.988 \times 10^{9} \frac{\text { Joule-meters }}{\text { Coulombs }^{2}} \\
& \mathrm{e}=\text { electron charge }=1.602 \times 10^{-19} \text { Coulombs }
\end{aligned}
$$




\begin{tabular}{|c|c|c|c|}
\hline The speed of light & $\mathbf{m}$ & $\mathbf{k}$ & $\mathbf{e}$ \\
\hline 299792458 & $(9109 * 4)$ & $(8988 * 250)$ & $(1602 * 185711)$ \\
\hline 299792458 & $(9109 * 4)$ & $(8988 * 517)$ & $(1602 * 184213)$ \\
\hline 299792458 & $(9109 * 4)$ & $(8988 * 784)$ & $(1602 * 182715)$ \\
\hline 299792458 & $(9109 * 4)$ & $(8988 * 1051)$ & $(1602 * 181217)$ \\
\hline 299792458 & $(9109 * 4)$ & $(8988 * 1318)$ & $(1602 * 179719)$ \\
\hline 299792458 & $(9109 * 4)$ & $(8988 * 1585)$ & $(1602 * 178221)$ \\
\hline 299792458 & $(9109 * 4)$ & $(8988 * 1852)$ & $(1602 * 176723)$ \\
\hline 299792458 & $(9109 * 4)$ & $(8988 * 2119)$ & $(1602 * 175225)$ \\
\hline 299792458 & $(9109 * 4)$ & $(8988 * 2386)$ & $(1602 * 173727)$ \\
\hline
\end{tabular}

$$
299792458=(9109 * 4)+(8988 * 250)+(1602 * 185711)
$$

etc.

\section{CONCLUSION}

Making a sequence of all phenomena in nature is conducted according to the exact mathematical laws (for such descriptions we can use theory of systems and cybernetics.) Results of this laws is codes 19, 7 and 931.

\section{References}

[1] L. Kurić, J. Comput Sci Biol 2 (2009) 101-116.

[2] L. Kurić, Journal de la Societe de statistique de Paris 127(2) (1986).

[3] L. Kurić, GJMR 10(1) (2010) 15.

[4] L. Kurić, Advances and Applications in Bioinformatics and Chemistry, 2010, 45-58.

[5] L. Kurić, GJMR 1(1) (2010) 15.

[6] L. Kurić, International Journal of Computer Technology and Application 2(2) (2011) 216-241.

[7] L. Kurić, International Journal of Computer Technology and Application 2(2) (2011) 258-273.

[8] L. Kurić, Journal of Chemical Engineering and Material Science 2(5) (2011). 
[9] Lutvo Kurić, International Letters of Chemistry, Physics and Astronomy 10 (2014) 62-73.

[10] Lutvo Kurić, International Letters of Chemistry, Physics and Astronomy 11(3) (2014) 202-213.

[11] Lutvo Kurić, International Letters of Chemistry, Physics and Astronomy 12 (2014) 31-50.

[12] Lutvo Kurić, International Letters of Chemistry, Physics and Astronomy 13(1) (2014) 11-20.

[13] Lutvo Kurić, International Letters of Chemistry, Physics and Astronomy 13(1) (2014) $42-53$.

[14] Lutvo Kurić, International Letters of Chemistry, Physics and Astronomy 13(2) (2014) 119-134.

[15] Lutvo Kurić, International Letters of Chemistry, Physics and Astronomy 13(2) (2014) 160-173.

[16] Lutvo Kurić, International Letters of Chemistry, Physics and Astronomy 13(2) (2014) 174-190.

[17] Lutvo Kurić, International Letters of Chemistry, Physics and Astronomy 14(1) (2014) 28-52. 